\title{
On Projectively Embeddable Complex-Foliated Structures
}

\author{
by
}

Takeo OHsAwA

\begin{abstract}
An extension of Kodaira's embedding theorem is proved for compact smoothly foliated manifolds with complex leaves. Existence of a leafwise positive line bundle is shown to be sufficient for $C^{k}$ projective embeddability for all $k<\infty$.
\end{abstract}

2010 Mathematics Subject Classification: Primary 32E40, 32V40; Secondary 53C40. Keywords: projective embedding, foliation, line bundle, curvature form $\bar{\partial}$-equation.

\section{Introduction}

A well known embedding theorem of Kodaira $[\mathrm{K}]$ says that every positive line bundle over a compact complex manifold is ample. Inspired by Matsushima's work $[\mathrm{M}]$ on the embedding of certain nonalgebraic tori by partially holomorphic maps, we would like to extend Kodaira's work to manifolds with partial complex structures as a continuation of [O-2].

For that, we shall focus our attention on an abstract question of embedding foliated manifolds with complex leaves into projective space.

Let $M$ be a paracompact $C^{\infty}$ manifold of dimension $2 n+r(n, r \in \mathbb{N})$ equipped with a $2 n$-dimensional foliation $\mathcal{F}$ such that the tangent bundle $\mathrm{T} \mathcal{F}$ of $\mathcal{F}$ has a complex structure $J \in \operatorname{EndT\mathcal {F}}\left(J^{2}=-\right.$ id) which induces integrable almost complex structures on the leaves of $\mathcal{F}$. Then $\mathcal{F}$ will be called an $n$-dimensional complex foliation on $M$.

Given such a pair $(M, \mathcal{F})$, one may consider $C^{k}$ complex vector bundles over $M$ whose transition functions are holomorphic along the leaves of $\mathcal{F}$. They will be called tangentially holomorphic vector bundles over $(M, \mathcal{F})$, or simply over $M$. Given a tangentially holomorphic vector bundle $E$ over $(M, \mathcal{F})$, a section $s$ of $E$

Communicated by M. Kashiwara. Received April 15, 2008. Revised October 3, 2011.

T. Ohsawa: Graduate School of Mathematics, Nagoya University,

Chikusaku Furocho, 464-8602 Nagoya, Japan;

e-mail: ohsawa@math.nagoya-u.ac.jp 
is called tangentially holomorphic if the restrictions of $s$ to the leaves of $\mathcal{F}$ are holomorphic. As usual, vector bundles of rank one are called line bundles.

Let $L \rightarrow M$ be a tangentially holomorphic $C^{\infty}$ line bundle equipped with a $C^{\infty}$ fiber metric $h$, let $D_{h}$ be a connection on $L$ compatible with $h$ and with complex structures of the leaves of $\mathcal{F}$, and let $\Theta_{h}$ be the curvature form of $h$. Recall that $\Theta_{h}$ is a 2-form on $M$ satisfying $D_{h}^{2} s=\Theta_{h} s$ for any $C^{2}$ section $s$ of $L$.

We say that $(L, h)$ is $\mathcal{F}$-positive if the restriction of $\sqrt{-1} \Theta_{h}$ to the leaves of $\mathcal{F}$ is everywhere positive as a real $(1,1)$-form.

The purpose of the present work is to establish the following.

Theorem 0.1. Let $M$ be a connected and compact $C^{\infty}$ manifold equipped with a complex foliation $\mathcal{F}$, and let $(L, h)$ be an $\mathcal{F}$-positive line bundle over $M$. Then, for any nonnegative integer $k$, there exists a positive integer $m_{0}$ such that, for any $m \geq m_{0}(m \in \mathbb{N})$, one can find $C^{k}$ tangentially holomorphic sections $s_{0}, \ldots, s_{N}$ of $L^{m}$ such that the ratio $\left(s_{0}: \cdots: s_{N}\right)$ is a $C^{k}$ embedding of $M$ into $\mathbb{C P}^{N}$. Here $\mathbb{C P}^{N}$ denotes the $N$-dimensional complex projective space.

It should be noted that a statement essentially equivalent to Theorem 0.1 was stated in [O-S] to be an application of a vanishing theorem of Kodaira type on Levi flat CR manifolds. The proof given there relies implicitly on the statement that the transverse regularity of the inverse of the tangential Laplacian is a consequence of a certain a priori estimate. However, it is hard to pinpoint an appropriate reference for that. Accordingly, our method in this article will be based on a completely different principle. The idea is to exploit the curvature condition to produce local solutions to the tangential Cauchy-Riemann equation with a good control of regularity and obtain a global solution by patching them together with the aid of successive approximations. This method was used by G. Tian [T] for the Cauchy-Riemann equation. It might be of interest that the method is robust under the addition of extra parameters.

\section{$\S 1$. Local theory}

We shall prepare a lemma on the parameter dependence of the canonical $L^{2}$ solutions of inhomogeneous Cauchy-Riemann equations in the local setting. It will eventually guarantee the existence of solutions of global tangential CauchyRiemann equations with values in sufficiently high tensor powers of a given $\mathcal{F}$ positive line bundle. The material presented here originates in [D-O].

The lemma will be formulated for $(0, q)$-forms although only the case of $(0,1)$ forms is needed afterwards. However, the general form may be useful for other purposes. 
Let $D$ be a pseudoconvex domain in $\mathbb{C}^{n}$ and let $U \subset \mathbb{R}^{r}$ be any convex domain. The coordinates of $\mathbb{C}^{n}$ and $\mathbb{R}^{r}$ will be denoted by $z=\left(z_{1}, \ldots, z_{n}\right)$ and $t=\left(t_{1}, \ldots, t_{r}\right)$, respectively.

We shall consider a family $v_{t}$ of $C^{\infty}(0, q)$-forms on $D$ depending smoothly on the parameter $t \in U$,

$$
v_{t}=\sum_{1 \leq i_{1}<\cdots<i_{q} \leq n} v_{i_{1} \ldots i_{q}}(z, t) d \bar{z}_{i_{1}} \wedge \cdots \wedge d \bar{z}_{i_{q}}
$$

for some complex-valued $C^{\infty}$ functions $v_{i_{1} \ldots i_{q}}$ on $D \times U$. The set of such $\left\{v_{t}\right\}_{t \in U}$ will be denoted by $C^{0, q}(D \times U)$. For simplicity the elements of $C^{0, q}(D \times U)$ will be denoted by $v_{t}$.

Let $C^{0, q}(D)$ denote the set of all $C^{\infty}(0, q)$-forms on $D$, and by $L_{(2)}^{0, q}(D$, loc $)$ the space of locally square integrable $(0, q)$-forms on $D$.

For any $v \in L_{(2)}^{0, q}(D$, loc $),|v|^{2}$ will stand for the function

$$
\sum_{1 \leq i_{1}<\cdots<i_{q} \leq n}\left|v_{i_{1} \ldots i_{q}}\right|^{2}
$$

where $v=\sum_{1 \leq i_{1}<\cdots<i_{q} \leq n} v_{i_{1} \ldots i_{q}} d \bar{z}_{i_{1}} \wedge \cdots \wedge d \bar{z}_{i_{q}}$. We put

$$
\left\langle v_{1}, v_{2}\right\rangle=\frac{1}{4}\left(\left|v_{1}+v_{2}\right|^{2}-\left|v_{1}-v_{2}\right|^{2}\right)+\frac{\sqrt{-1}}{4}\left(\left|v_{1}+\sqrt{-1} v_{2}\right|^{2}-\left|v_{1}-\sqrt{-1} v_{2}\right|^{2}\right)
$$

for $v_{1}, v_{2} \in L_{(2)}^{0, q}(D, \mathrm{loc})$.

Further, we put

$$
C_{0}^{0, q}(D)=\left\{v \in C^{0, q}(D) \mid \operatorname{supp} v \text { is compact }\right\} .
$$

We denote by $\bar{\partial}$ the complex exterior differentiation of type $(0,1)$. The same symbol $\bar{\partial}$ will also stand for the operator from $C^{0, q}(D \times U)$ to $C^{0, q+1}(D \times U)$ defined by $v_{t} \mapsto \bar{\partial} v_{t}$.

Let us recall a basic result of Hörmander.

Theorem 1.1 (cf. [H, Theorem 2.2.1']). Let $D$ be a pseudoconvex domain in $\mathbb{C}^{n}$, let $\psi$ be plurisubharmonic in $D$ and let $\kappa$ be a real-valued continuous function such that the difference

$$
\sum_{j, k=1}^{n} \frac{\partial^{2} \psi}{\partial z_{j} \partial \bar{z}_{k}} \xi_{j} \bar{\xi}_{k}-e^{\kappa} \sum_{j=1}^{n}\left|\xi_{j}\right|^{2}
$$

is a positive measure for arbitrary complex numbers $\xi_{j}$. Then, for every $v \in$ $L_{(2)}^{0, q}(D, \mathrm{loc}), q>0$, such that $\bar{\partial} v=0$ and

$$
\int_{D}|v|^{2} e^{-(\psi+\kappa)} d \lambda<\infty,
$$


one can find a form $u \in L_{(2)}^{0, q-1}(D$, loc $)$ such that $\bar{\partial} u=v$ and

$$
q \int_{D}|u|^{2} e^{-\psi} d \lambda \leq \int_{D}|v|^{2} e^{-(\psi+\kappa)} d \lambda
$$

Here $d \lambda$ denotes the Lebesgue measure on $\mathbb{C}^{n}$.

Once for all we fix a $C^{\infty}$ real-valued function $\varphi=\varphi(z, t)$ on $D \times U$. We shall denote by $L_{(2), t}^{0, q}(D)$ the completion of the space $C_{0}^{0, q}(D)$ with respect to the inner product

$$
\left(v_{1}, v_{2}\right)_{t}=\int_{D} e^{-\varphi(\cdot, t)}\left\langle v_{1}, v_{2}\right\rangle d \lambda .
$$

We put $\|v\|_{t}=(v, v)_{t}^{1 / 2}$.

By an abuse of notation, $\bar{\partial}$ will also stand for the closed operator from $L_{(2), t}^{0, q}(D)$ to $L_{(2), t}^{0, q+1}(D)$ whose domain of definition is

$$
\operatorname{Dom} \bar{\partial}=\left\{u \in L_{(2), t}^{0, q}(D) \mid \bar{\partial} u \in L_{(2), t}^{0, q+1}(D)\right\} .
$$

The adjoint of the operator $\bar{\partial}: L_{(2), t}^{0, q}(D) \rightarrow L_{(2), t}^{0, q+1}(D)$ is denoted by $\bar{\partial}_{t}^{*}$.

From Theorem 1.1, it is easy to deduce the following.

Proposition 1.1. Assume that $D$ is bounded and that $\varphi(z, t)-m|z|^{2}$ is plurisubharmonic in $z$ for some $m>0$. Then there exists a constant $C$, independent of $\varphi$, $t$ and $m$, such that, for any $f_{t} \in L_{(2), t}^{0, q}(D), q>0$, there exist $g_{t} \in \operatorname{Ker} \bar{\partial} L_{(2), t}^{0, q}(D)$ and $h_{t} \in \operatorname{Dom} \bar{\partial}_{t}^{*} \subset L_{(2), t}^{0, q+1}(D)$ satisfying

$$
f_{t}=g_{t}+\bar{\partial}_{t}^{*} h_{t}, \quad\left\|g_{t}\right\|_{t} \leq\left\|f_{t}\right\|_{t} \quad \text { and } \quad m\left\|h_{t}\right\|_{t}^{2} \leq C\left\|f_{t}\right\|_{t}^{2} .
$$

Proof. By Theorem 1.1, there exists a constant $C$, independent of $\varphi, t$ and $m$, such that, for any $v_{t} \in \operatorname{Ker} \bar{\partial} \cap L_{(2), t}^{0, q}(D), q>0$, there exist $u_{t} \in \operatorname{Dom} \bar{\partial}_{t} \subset L_{(2), t}^{0, q-1}(D)$ satisfying

$$
v_{t}=\bar{\partial} u_{t} \quad \text { and } \quad m\left\|u_{t}\right\|_{t}^{2} \leq C\left\|v_{t}\right\|_{t}^{2} .
$$

Therefore, the image of $\bar{\partial}_{t}^{*}$ in $L_{(2), t}^{0, q}(D)$ is closed for such $C$, and, for any $k_{t} \in$ $\operatorname{Im} \bar{\partial}_{t}^{*} \subset L_{(2), t}^{0, q}(D), q \geq 0$, there exist $h_{t} \in \operatorname{Dom} \bar{\partial}_{t}^{*} \subset L_{(2), t}^{0, q+1}(D)$ satisfying

$$
k_{t}=\bar{\partial}_{t}^{*} h_{t} \quad \text { and } \quad m\left\|h_{t}\right\|_{t}^{2} \leq C\left\|k_{t}\right\|_{t}^{2} .
$$

The desired conclusion follows from this and the orthogonal decomposition

$$
L_{(2), t}^{0, q}(D)=\operatorname{Ker} \bar{\partial} \oplus \operatorname{Im} \bar{\partial}_{t}^{*} .
$$

Let $D, \varphi, m$ and $C$ be as in Proposition 1.1. Given any $v_{t} \in C^{0, q}(D \times U) \cap$ $\operatorname{Ker} \bar{\partial}, q>0$, satisfying $\sup _{t \in U}\left\|v_{t}\right\|_{t}<\infty$, we then have a uniquely defined family 
$u_{t} \in L_{(2), t}^{0, q-1}(D)$ such that $\bar{\partial} u_{t}=v_{t}, m\left\|u_{t}\right\|_{t}^{2} \leq C\left\|v_{t}\right\|_{t}^{2}$ and $u_{t} \perp \operatorname{Ker} \bar{\partial}$ in $L_{(2), t}^{0, q-1}(D)$, by Theorem 1.1.

Lemma 1.1. Let $v_{t}$ and $u_{t}$ be as above. Suppose moreover that

$$
\begin{aligned}
& C_{1}:=\sup _{D \times U} \max _{j}\left|\frac{\partial \varphi}{\partial t_{j}}\right|<\infty, \\
& C_{2}:=\sup _{D \times U} \max _{i, j}\left|\frac{\partial^{2} \varphi}{\partial \bar{z}_{i} \partial t_{j}}\right|<\infty, \\
& C_{3}:=\sup _{U}\left\|v_{t}\right\|_{t}<\infty, \\
& C_{4}:=\sup _{U} \max _{i}\left\|\frac{\partial v_{t}}{\partial t_{i}}\right\|_{t}<\infty .
\end{aligned}
$$

Then, for any $t, t_{0} \in U$ and $f \in L_{(2), t}^{0, q-1}(D)$,

$$
\sqrt{m}\left|\left(u_{t}, f\right)_{t}-\left(u_{t_{0}}, f\right)_{t_{0}}\right| \leq \sqrt{C} e^{C_{1}}\left(C_{1}+C_{2}+C_{1} C_{3}+C_{4}\right)\|f\|_{t_{0}}\left|t-t_{0}\right| .
$$

Proof. By Proposition 1.1,

$$
f=g+\bar{\partial}_{t_{0}}^{*} h, \quad g \in \operatorname{Ker} \bar{\partial} .
$$

Then

$$
\left(u_{t}, f\right)_{t}=\left(u_{t}, g\right)_{t}+\left(u_{t}, \bar{\partial}_{t_{0}}^{*} h\right)_{t} .
$$

Since $u_{t} \perp \operatorname{Ker} \bar{\partial}$ in $L_{(2), t}^{0, q-1}(D)$, we have

$$
\left(u_{t}, g\right)_{t}=0 \quad \text { for all } t .
$$

On the other hand,

$$
\begin{aligned}
\left(u_{t}, \bar{\partial}_{t_{0}}^{*} h\right)_{t} & =\left(e^{-\varphi(\cdot, t)+\varphi\left(\cdot, t_{0}\right)} u_{t}, \bar{\partial}_{t_{0}}^{*} h\right)_{t_{0}} \\
& =\left(\bar{\partial}\left(\varphi\left(\cdot, t_{0}\right)-\varphi(\cdot, t)\right) u_{t}, h\right)_{t}+\left(e^{-\varphi(\cdot, t)+\varphi\left(\cdot, t_{0}\right)} v_{t}, h\right)_{t_{0}} \\
\left(u_{t_{0}}, f\right)_{t_{0}} & =\left(v_{t_{0}}, h\right)_{t_{0}} .
\end{aligned}
$$

Combining (1.2)-(1.4) with $m\left\|u_{t}\right\|_{t}^{2} \leq C\left\|v_{t}\right\|_{t}^{2}$ and $m\|h\|_{t_{0}}^{2} \leq C\|f\|_{t_{0}}^{2}$ we obtain (1.1).

Since Proposition 1.1 similarly implies that $\sqrt{m}\left\|u_{t}-u_{t_{0}}\right\|_{t} \leq C^{\prime}\left|t-t_{0}\right|$ with a constant $C^{\prime}$ independent of $m$, we infer from (1.3) that

$$
\begin{aligned}
\left(u_{t}, \bar{\partial}_{t_{0}}^{*} h\right)_{t}= & \left(\bar{\partial}\left(\varphi\left(\cdot, t_{0}\right)-\varphi(\cdot, t)\right) u_{t_{0}}, h\right)_{t}+\left(e^{-\varphi(\cdot, t)+\varphi\left(\cdot, t_{0}\right)} v_{t}, h\right)_{t_{0}} \\
& +O\left(\left|t-t_{0}\right|^{2}\right) .
\end{aligned}
$$

Hence $\left(u_{t}, f\right)_{t}$ is of class $C^{1}$ on $U$. 
Then we note that the conditions

$$
\bar{\partial} u_{t}=v_{t}, \quad \bar{\partial}_{t}^{*} u_{t}=0 \quad \text { and } \quad m\left\|u_{t}\right\|_{t}^{2} \leq C\left\|v_{t}\right\|_{t}^{2},
$$

for $v_{t} \in C^{0, q}(D \times U)$, imply that $u_{t}$ is equicontinuous as a family of $(0, q)$-forms on every compact subset of $D$. Since $\left(u_{t}, f\right)_{t}$ is continuous for any $f$, the equicontinuity of $u_{t}$ must imply the continuity of $u_{t}$ on $D \times U$. Otherwise $\left(u_{t}, f\right)_{t}$ would not be continuous for some $f$.

Similarly, since $\left(u_{t}, f\right)_{t}$ is $C^{1}$ for any $f, u_{t}$ must be $C^{1}$ on $D \times U$.

Moreover, from the equation

$$
\frac{\partial}{\partial t_{j}}\left(u_{t}, f\right)_{t}=\left(\frac{\partial u_{t}}{\partial t_{j}}, f\right)_{t}-\left(\frac{\partial \varphi(\cdot, t)}{\partial t_{j}} u_{t}, f\right)_{t}
$$

which holds whenever $f$ is compactly supported, and from the estimate (1.1), it is easy to see that $\frac{\partial u_{t}}{\partial t_{j}}\left(t_{0}\right) \in L_{(2), t_{0}}^{0, q-1}(D), 1 \leq j \leq r$. Therefore

$$
\begin{aligned}
& \left(u_{t}, \bar{\partial}_{t_{0}}^{*} h\right)_{t} \\
& =\left(\bar{\partial}\left(\varphi\left(\cdot, t_{0}\right)-\varphi(\cdot, t)\right)\left(u_{t_{0}}+\sum_{j=1}^{r} \frac{\partial u_{t}}{\partial t_{j}}\left(t_{0}\right)\left(t_{j}-t_{0 j}\right)\right), h\right)_{t}+\left(v_{t}, h\right)_{t} \\
& \quad+O\left(\left|t-t_{0}\right|^{3}\right)
\end{aligned}
$$

which implies that $\left(u_{t}, f\right)_{t}$ is of class $C^{2}$ on $U$.

Hence $u_{t}$ is $C^{2}$ on $D \times U$, provided that the derivatives of $\varphi$ and $\bar{\partial} \varphi$ up to the second order are bounded on $D \times U$ and those of $v_{t}$ are square integrable uniformly in $t$. Hence, taking into account the dependence of the estimates on $m$ as in (1.1), we obtain the following by induction.

Proposition 1.2. Let $v_{t}$ and $u_{t}$ be as before. Suppose moreover that $\nabla_{t}^{i} v_{t}$ are square integrable for $0 \leq i \leq k$ and that $\left|\nabla_{t}^{j} \varphi\right|$ are $\left|\nabla_{t}^{j} \bar{\partial} \varphi\right|$ are bounded by a constant $C_{(k)}$ on $D \times U$ for $1 \leq j \leq k$. Here $\nabla_{t}$ denotes the gradient in $t$ with respect to the coordinates $(z, t)$, and $C_{(k)}$ is independent of $m$. Then $u_{t}$ is of class $C^{k}$ on $D \times U$. Moreover there exists a constant $C_{\langle k\rangle}$, depending on $C_{(k)}$ but not on $m$, such that

$$
\sqrt{m}\left\|u_{t}\right\|_{t, k} \leq C_{\langle k\rangle}\left\|v_{t}\right\|_{t, k},
$$

where

$$
\|w\|_{t, k}=\sum_{j=0}^{k}\left\|\nabla_{t}^{j} w\right\|_{t}
$$


We need later an elementary observation on the modified norms

$$
\|w\|_{t, k}^{\prime}=\sum_{j=0}^{k} c_{j}\left\|\nabla_{t}^{j} w\right\|_{t}
$$

and

$$
\|w\|_{t, k, b}^{\prime}=\sum_{j=0}^{k} c_{j}\left\|b \nabla_{t}^{j} w\right\|_{t}
$$

for $c_{0}=1>c_{1}>\cdots>c_{k}>0$ and a nowhere vanishing $C^{\infty}$ function $b=b(z, t)$ on $D \times U$.

Note that Proposition 1.2 holds for the norms \|\|$_{t, k}^{\prime}$, with the same constant $C_{\langle k\rangle}$, independently of the choice of $c_{j}(1 \leq j \leq k)$.

For simplicity, we assume that $D$ and $U$ are bounded domains, and $\varphi$ and $b$ are defined on a neighbourhood of the closure $\overline{D \times U}$ of $D \times U$.

Lemma 1.2. In the above situation, let $l \in \mathbb{N}$ and let $b_{\mu}(z, t)(\mu=1, \ldots, l)$ be nowhere vanishing $C^{\infty}$ functions on $D \times U$. Then, for any $k \in \mathbb{N}$, there exists $c>0$ such that, for any elements $v_{\mu, t}(\mu=1, \ldots, l)$ of $C^{0, q}(D \times U)$ which are smoothly extendable to a neighbourhood of $\overline{D \times U}$, the estimate

$$
\sum_{\mu=1}^{l}\left\|b_{\mu}(z, t) v_{\mu, t}\right\|_{t, k}^{\prime} \leq 2 \sum_{\mu=1}^{l}\left\|v_{\mu, t}\right\|_{t, k, b_{\mu}}^{\prime}
$$

holds for $c_{j}=c^{-j}(j=0,1, \ldots, k)$.

The proof is straightforward and may well be left to the reader.

\section{$\S 2$. Proof of Theorem 0.1}

Let $(M, \mathcal{F})$ and $(L, h)$ be as in the assumption. Let $x \in M$ be any point, let $\rho>0$, and let $B$ be a neighbourhood of $x$ on which local coordinates $(z, t)=$ $\left(z_{1}, \ldots, z_{n}, t_{1}, \ldots, t_{r}\right)$ are defined in such a way that $x$ is mapped by $(z, t)$ to $(0,0)$, and $B$ is mapped onto $\mathbb{D}(\rho)^{n} \times(\mathbb{D}(1) \cap \mathbb{R})^{r}, \mathbb{D}(\rho)=\{\zeta \in \mathbb{C}|| \zeta \mid<\rho\}$, holomorphically in $z$ with $X t=0$ for any $X \in T \mathcal{F}$.

Shrinking $B$ if necessary, we may assume that there exists a nowhere vanishing $C^{\infty}$ tangentially holomorphic section of $L$ over $B$, say $s$.

For any section $\sigma$ of $L$, let $|\sigma|_{h}$ denote the length of $\sigma$ with respect to $h$, and let $\gamma$ be the real-valued $C^{\infty}$ function on $B$ defined by

$$
|\sigma|_{h}^{2}=e^{-\gamma}|\sigma / s|^{2} \quad \text { on } B \text { for all } \sigma
$$


Let

$$
\begin{aligned}
\gamma(= & \gamma(z, t)) \\
= & 2 \operatorname{Re}\left(a(t)+\sum_{i=1}^{n} a_{i}(t) z_{i}+\sum_{i, j=1}^{n} a_{i j}(t) z_{i} z_{j}+\sum_{i, j, k} a_{i j k}(t) z_{i} z_{j} z_{k}\right) \\
& +\sum_{i, j=1}^{n} b_{i j}(t) z_{i} \bar{z}_{j}+2 \operatorname{Re} \sum_{i, j, k} c_{i j k}(t) z_{i} \bar{z}_{j} \bar{z}_{k}+O\left(|z|^{4}\right)
\end{aligned}
$$

be the Taylor expansion of $\gamma$ around $x$. Replacing $s$ by

$$
s^{\prime}=s \cdot \exp \left(a(t)+\sum_{i=1}^{n} a_{i}(t) z_{i}+\sum_{i, j=1}^{n} a_{i j}(t) z_{i} z_{j}+\sum_{i, j, k} a_{i j k}(t) z_{i} z_{j} z_{k}\right)
$$

we may assume that (2.1) is of the form

$$
\gamma=\sum_{i, j} b_{i j}(t) z_{i} \bar{z}_{j}+2 \operatorname{Re} \sum_{i, j, k} c_{i j k}(t) z_{i} \bar{z}_{j} \bar{z}_{k}+O\left(|z|^{4}\right) .
$$

Further, since $(L, h)$ is $\mathcal{F}$-positive, $\left(b_{i j}(t)\right)$ is everywhere positive definite. Therefore, by a coordinate change, we may assume that

$$
\gamma=\sum_{i=1}^{n}\left|z_{i}\right|^{2}+O\left(|z|^{4}\right)
$$

where the original radius $\rho$ should be replaced by a possibly smaller positive number, say $\rho^{\prime}$.

If (2.3) is satisfied, we shall say that $s^{\prime}$ is a distinguished coordinate (associated to $\left.\rho^{\prime}\right)$. The constant $\rho^{\prime}$ will be referred to as a distinguished radius.

Moreover we put in this situation

$$
\begin{aligned}
\Omega_{m} & =\left\{\left.(z, t)|m| z\right|^{3}<\rho^{\prime 3},|t|<1\right\}, \\
D_{m} & =\left\{\left.z|2 m| z\right|^{3}<\rho^{\prime 3}\right\}, \\
U_{c^{\prime}} & =\left\{t\left|c^{\prime}\right| t \mid<1\right\} \quad\left(c^{\prime} \geq 1\right) .
\end{aligned}
$$

Then, in view of Proposition 1.2 and a remark after it, we infer from (2.3) the following. (See also [Dm, p. 270, Proposition 12.10].)

Sublemma 2.1. For any $k \in \mathbb{N}$ and $c^{\prime} \geq 1$, there exists a constant $C_{[k]}$ such that, for any $m \in \mathbb{N}$ and for any $v_{t} \in C^{0, q}\left(\Omega_{m}\right) \cap \operatorname{Ker} \bar{\partial}, q>0$, one can find $u_{t} \in$ $C^{0, q-1}\left(D_{m} \times U_{c^{\prime}}\right)$ satisfying $\bar{\partial} u_{t}=v_{t}$ on $D_{m} \times U_{c^{\prime}}$ and $\sqrt{m}\left\|u_{t}\right\|_{t, k}^{\prime} \leq C_{[k]}\left\|v_{t}\right\|_{t, k}^{\prime}$.

For each distinguished coordinate $\left(z_{x}, t_{x}\right)$, we shall denote by $I_{m, x}$ and $J_{m, x}$ the neighbourhoods of $x$ that are identified with $D_{m} \times U_{3 / 2}$ and $D_{2 m} \times U_{2}$, respectively. 
It is clear that, for any point $x_{0} \in M$, there exists a neighbourhood $V \ni x_{0}$ such that one has a $C^{\infty}$ family of distinguished frames and associated distinguished coordinates around $x \in V$, parametrized by $x \in V$. Hence, since $M$ is compact, we have the following.

Sublemma 2.2. There exist $\nu \in \mathbb{N}$ and $C: \mathbb{N} \rightarrow(0, \infty)$ such that, for any $k, m \in \mathbb{N}$ one can find $N \in \mathbb{N}, x_{m 1}, \ldots, x_{m N} \in M$ and distinguished coordinates $\left(z_{(m \alpha)}, t_{(m \alpha)}\right)(1 \leq \alpha \leq N)$ around $x_{m \alpha}$, with distinguished radius $\rho_{0}$ independent of $m$, such that

$$
\begin{aligned}
& \left|\nabla_{\left.t_{(m \alpha}\right)}^{k} t_{(m \beta)}\right|<C(k) \quad \text { on } I_{m, x_{m \alpha}} \cap I_{m, x_{m \beta}}, \\
& M=\bigcup_{\alpha=1}^{N} J_{m, x_{m \alpha}}, \\
& \bigcap_{\kappa=1}^{\mu} I_{m, x_{m \alpha_{\kappa}}}=\emptyset \quad \text { if } \alpha_{1}<\cdots<\alpha_{\mu} \text { and } \mu>\nu .
\end{aligned}
$$

In the above situation, we shall call $\left\{J_{m, x_{m \alpha}}\right\}_{1 \leq \alpha \leq N}$ a $(k, m)$-filamental covering of $M$.

With these preparations, we construct tangentially holomorphic $C^{k}$ sections of $L^{m}$ as follows.

Let $x \in M$ be any point, let $s$ be a distinguished frame and let $(z, t)$ be distinguished coordinates around $x$ associated to $s$. Given any $C^{\infty}$ tangentially holomorphic function on $I_{m, x}$, we are going to show that there exist, for any $k \in \mathbb{N}$ and for any $m \gg k$, a tangentially holomorphic $C^{k}$ section $\sigma_{m}$ of $L^{m}$ over $M$ satisfying

$$
\begin{array}{r}
\left|f-\sigma_{m} / s^{m}\right|_{x}<1 / m, \\
\left|d f-d\left(\sigma_{m} / s^{m}\right)\right|_{x}<1 / m .
\end{array}
$$

Here the norm of $d f-d\left(\sigma_{m} / s^{m}\right)$ is measured with respect to the metric $\sum d z_{i} d \bar{z}_{i}+$ $\sum d t_{j}^{2}$

For that, we first fix $(k, m)$-filamental coverings $\left\{J_{m, x_{m \alpha}}\right\}_{1 \leq \alpha \leq N(m)}(m \in \mathbb{N})$ of $M$ with $x_{m 1}=x$ for all $m$. Let $s_{m \alpha}$ be the associated distinguished frames around $x_{m \alpha}$ such that $s_{m 1}=s$ for all $m$.

Let $\mathcal{X}: \mathbb{R} \rightarrow \mathbb{R}$ be a nonnegative $C^{\infty}$ function satisfying $\mathcal{X}(t)=1$ on $(-\infty, 1]$ and $\operatorname{supp} \mathcal{X} \cap[2, \infty)=\emptyset$. We put

$$
\mathcal{X}_{m \alpha}\left(z_{(m \alpha)}, t_{(m \alpha)}\right)=\mathcal{X}\left(4\left|z_{(m \alpha)}\right|^{3} / \rho_{0}^{3}\right) \mathcal{X}\left(2\left|t_{(m \alpha)}\right|\right)
$$


By extending $\mathcal{X}_{m \alpha}$ as 0 outside the set $\left\{\left|z_{(m \alpha)}\right|<\rho_{0},\left|t_{(m \alpha)}\right|<1\right\}$, we obtain $C^{\infty}$ functions $\widetilde{\mathcal{X}}_{m \alpha}$ on $M$. Then we put

$$
\eta_{m \alpha}=\widetilde{\mathcal{X}}_{m \alpha} / \sum_{\beta=1}^{N(m)} \widetilde{\mathcal{X}}_{m \beta} .
$$

The system $\eta_{m \alpha}$ is then a $C^{\infty}$ partition of unity satisfying $\operatorname{supp} \eta_{m \alpha} \subset I_{m, x_{m \alpha}}$.

We put

$$
\tilde{f}=\left\{\begin{array}{ll}
\mathcal{X}_{m 1} f s^{m} & \text { on } I_{m, x}, \\
0 & \text { otherwise }
\end{array} \quad v_{m}=\bar{\partial} \tilde{f},\right.
$$

and define $v_{m \alpha}$ by

$$
v_{m \alpha} s_{m \alpha}^{m}=v_{m} \quad \text { on } I_{m, x_{m \alpha}},
$$

where $\bar{\partial}$ is defined leafwise as before.

Obviously, one may choose the frames $s_{m \alpha}$ and coordinates $\left(z_{(m \alpha)}, t_{(m \alpha)}\right)$ in such a way that, for any fixed $k$, there exist $\varepsilon_{k}>0$ and $m_{0}$ such that, for any $m>m_{0}$,

$$
\sum_{\alpha=1}^{N(m)}\left\|v_{m \alpha}\right\|_{t_{(m \alpha)}, k}^{\prime} \leq e^{-m^{1 / 6}}
$$

for $c_{j}=\varepsilon_{k}^{j} m^{-j}(0 \leq j \leq k)$. From now on, $c_{j}$ will be chosen in that way.

By Sublemma 2.1, one can find $u_{m \alpha} \in C^{0,0}\left(J_{m, x_{m \alpha}}\right)$ satisfying $\bar{\partial} u_{m \alpha}=v_{m \alpha}$ on $J_{m, x_{m \alpha}}$ and

$$
\sqrt{m}\left\|u_{m \alpha}\right\|_{t_{(m \alpha)}, k}^{\prime} \leq C_{[k]}\left\|v_{m \alpha}\right\|_{t_{(m \alpha)}, k}^{\prime} .
$$

We put

$$
\widetilde{u}_{m}=\sum_{\alpha} \eta_{m \alpha} s_{m \alpha}^{m} u_{m \alpha} \quad \text { and } \quad \widetilde{u}_{m \alpha}=\widetilde{u}_{m} / s_{m \alpha}^{m} \quad \text { on } I_{m, x_{m \alpha}} .
$$

Since $\bar{\partial} \eta_{m \alpha}$ is at most of order $m^{1 / 3}$, we obtain, in view of Lemma 1.2 and the strong ellipticity of the Laplacian along $\mathcal{F}$, the estimates

$$
\begin{aligned}
\sum_{\alpha}\left\|\widetilde{u}_{m \alpha}\right\|_{t_{(m \alpha)}, k}^{\prime} & \leq 2 \nu \sum_{\alpha}\left\|u_{m \alpha}\right\|_{t_{(m \alpha)}, k}^{\prime}, \\
\sum_{\alpha}\left\|v_{m \alpha}-\bar{\partial} \widetilde{u}_{m \alpha}\right\|_{t_{(m \alpha)}, k}^{\prime} & \leq 2 \nu m^{1 / 3} \sum_{\beta}\left\|u_{m \beta}\right\|_{t_{(m \beta)}, k}^{\prime},
\end{aligned}
$$

on replacing $\varepsilon_{k}$ by a smaller number if necessary. 
Combining (2.12) with (2.9) and (2.10), we obtain

$$
\sum_{\alpha}\left\|v_{m \alpha}-\bar{\partial} \widetilde{u}_{(m \alpha)}\right\|_{t_{(m \alpha)}, k}^{\prime} \leq \widetilde{C}_{k} m^{-1 / 6}
$$

for some constant $C_{k}$ independent of $m$.

Therefore, for any $k$, by choosing $m$ sufficiently large and $\varepsilon_{k}$ sufficiently small, we have, in view of (2.10) and (2.11),

$$
\begin{aligned}
\sum_{\alpha}\left\|\widetilde{u}_{m \alpha}\right\|_{t_{(m \alpha)}, k}^{\prime} & \leq \frac{1}{2} \sum_{\alpha}\left\|v_{m \alpha}\right\|_{t_{(m \alpha)}, k}^{\prime}, \\
\sum_{\alpha}\left\|v_{m \alpha}-\bar{\partial} \widetilde{u}_{m \alpha}\right\|_{t_{(m \alpha)}, k}^{\prime} & \leq \frac{1}{2} \sum_{\alpha}\left\|v_{m \alpha}\right\|_{t_{(m \alpha)}, k}^{\prime} .
\end{aligned}
$$

Hence one can repeat this procedure, with a fixed choice of $\varepsilon_{k}$, to obtain for $m \gg 1$ a system of infinite series

$$
\left\{\sum_{\kappa=0}^{\infty} \widetilde{u}_{m \alpha, \kappa}\right\}_{1 \leq \alpha \leq N(m)}
$$

such that $\sum_{\kappa=0}^{\infty} \widetilde{u}_{m \alpha, \kappa}$ is convergent with respect to \|\|$_{t_{(m \alpha)}, k}^{\prime}$,

$$
\begin{aligned}
& s_{m \alpha}^{m} \widetilde{u}_{m \alpha, \kappa}=s_{m \beta}^{m} \widetilde{u}_{m \beta, \kappa} \quad \text { on } I_{m, x_{m \alpha}} \cap I_{m, x_{m \beta}}, \\
& \bar{\partial}\left(\sum_{\kappa=0}^{\infty} \widetilde{u}_{m \alpha, \kappa}\right)=v_{m \alpha} \quad \text { on } I_{m, x_{m \alpha}}, \\
& \left\|\sum_{\kappa=0}^{\infty} \widetilde{u}_{m \alpha, \kappa}\right\|_{t_{(m \alpha), k}}^{\prime} \leq e^{-m^{1 / 7} .}
\end{aligned}
$$

In particular, we obtain from (2.18) the $C^{k}$ differentiability of $\sum_{\kappa=0}^{\infty} \widetilde{u}_{m \alpha, \kappa}$.

Moreover, by (2.9), (2.3) and Cauchy's estimate along $\mathcal{F}$, we have

$$
\left|f-\left(\widetilde{f}-\sum_{\kappa=0}^{\infty} \widetilde{u}_{m 1, \kappa}\right)\right|_{x} \leq e^{-m^{1 / 8}}, \quad\left|d f-d\left(\widetilde{f}-\sum_{\kappa=0}^{\infty} \widetilde{u}_{m 1, \kappa}\right)\right|_{x} \leq e^{-m^{1 / 8}},
$$

for $m \gg 1$. Hence, $\sigma_{m}:=f-s_{m \alpha}^{m} \sum_{\kappa=0}^{\infty} \widetilde{u}_{m \alpha, \kappa}$ satisfies the requirements (2.7) and (2.8) for $m \gg 1$.

Taking the components of a local coordinate system around $x$ as $f$, we obtain tangentially holomorphic $C^{k}$ sections $s_{1}, \ldots, s_{n+r}$ of $L^{m}$ such that $\left(s_{1} / s_{m 1}^{m}, \ldots, s_{n+r} / s_{m 1}^{m}\right)$ embeds a neighbourhood of $x$ into $\mathbb{C}^{n+r}$.

Similarly, for any two distinct points $x$ and $y$ of $M$, one can find tangentially holomorphic $C^{k}$ sections $s, s^{\prime}$ of $L^{m}$ satisfying $\left(s(x): s^{\prime}(x)\right) \neq\left(s(y): s^{\prime}(y)\right)$. 
Thus, by the usual compactness argument, one can find tangentially holomorphic $C^{k}$ sections $s_{0}, \ldots, s_{N}$ of $L^{m}$ for $m \gg 1$ such that $\left(s_{0}: \cdots: s_{N}\right)$ is a $C^{k}$ embedding of $M$ into $\mathbb{C P}^{N}$.

\section{$\S 3$. Notes and remarks}

Since every $C^{k}$-embeddable complex-foliated manifold $(M, \mathcal{F})$ admits a $C^{k} \mathcal{F}$ positive line bundle (e.g. the restriction of the hyperplane section bundle) if $k \geq 2$, it is obvious from the proof of Theorem 0.1 that the following refined statement is also true.

Theorem 3.1. Let $M$ be a compact $C^{\infty}$ manifold and let $\mathcal{F}$ be a $C^{\infty}$ foliation on $M$ whose leaves are complex. Then $(M, \mathcal{F})$ admits a $C^{k}$ tangentially holomorphic embedding into some $\mathbb{C P}^{N}$ for any $k \in \mathbb{N}$ if and only if there exists a $C^{k}$ $\mathcal{F}$-positive line bundle over $M$ for any $k \in \mathbb{N}$.

Corollary 3.1. Let $M$ be a compact $C^{\infty}$ Riemannian manifold with an oriented 2-dimensional $C^{\infty}$ foliation $\mathcal{F}$. Then there exist $C^{k}$ embeddings $(k \in \mathbb{N})$ into some $\mathbb{C P}^{N}$ which are conformal along the leaves of $\mathcal{F}$ if and only if there exists a closed $C^{1}$ submanifold of $M$ which intersects with every leaf of $\mathcal{F}$ transversally (along a nonempty set).

It is also easy to see that the method of the proof of Theorem 0.1 can be directly applied to prove the $C^{k}$-solvability of tangential Cauchy-Riemann equations of more general type.

Let $T^{1,0} \mathcal{F}$ (resp. $T^{0,1} \mathcal{F}$ ) denote the subbundle of $T \mathcal{F} \otimes \mathbb{C}$ consisting of holomorphic (resp. antiholomorphic) vectors, and let $\left.\left(T^{1,0} \mathcal{F}\right)^{*}\right)\left(\operatorname{resp} .\left(T^{0,1} \mathcal{F}\right)^{*}\right)$ be its dual.

Given a tangentially holomorphic vector bundle $E \rightarrow M$ of class $C^{k}$, we denote by $C_{(k), \mathcal{F}}^{p, q}(E)$ the set of $C^{k}$ sections of $E \otimes \bigwedge^{p}\left(T^{1,0} \mathcal{F}\right)^{*} \otimes \bigwedge^{q}\left(T^{0,1} \mathcal{F}\right)^{*}$ over $M$ that are $C^{\infty}$ along $\mathcal{F}$, and let $\bar{\partial}: C_{(k), \mathcal{F}}^{p, q}(E) \rightarrow C_{(k), \mathcal{F}}^{p, q+1}(E)$ denote the complex exterior differentiation of type $(0,1)$ along $\mathcal{F}$. Then the method in $\S 2$ entails

Theorem 3.2. Let $(M, \mathcal{F})$ be as in Theorem 3.1, and let $L \rightarrow M$ be an $\mathcal{F}$-positive $C^{\infty}$ line bundle. Then, for any tangentially holomorphic $C^{k}$ vector bundle $E \rightarrow M$ there exists $m_{0} \in \mathbb{N}$ such that, for any $m \geq m_{0}$ and for any $v \in C_{(k), \mathcal{F}}^{p, q}\left(E \otimes L^{m}\right) \cap$ Ker $\bar{\partial}, q>0$, one can find a $u \in C_{(k), \mathcal{F}}^{p, q-1}\left(E \otimes L^{m}\right)$ satisfying $\bar{\partial} u=v$.

It should be noted that the statement of Theorem 3.2 becomes false if $k=\infty$ (cf. $[\mathrm{O}-1]$ ). 


\section{Acknowledgements}

The author is grateful to the referee for the useful comments.

After finishing the first draft of the manuscript, the author was informed by S. Frankel that a similar embeddability criterion had been considered for complex laminations in M. Gromov's paper [G, pp. 401-402] with an indication on how to prove it. For a complete proof of Gromov's result, see [D]. The author thanks M. Tsukamoto for useful information on Gromov's article. He also thanks M. Adachi for pointing out a mistake before the proofreading.

\section{References}

[Dm] J.-P. Demailly, Complex analytic and differential geometry, manuscript.

[D] B. Deroin, Laminations dans les espaces projectifs complexes, J. Inst. Math. Jussieu 7 (2008), 67-91. Zbl 1153.32004 MR 2398147

[D-O] K. Diederich and T. Ohsawa, On the parameter dependence of solutions to the $\bar{\partial}-$ equation, Math. Ann. 289 (1991), 581-587. Zbl 0789.35119 MR 1103037

[F] S. Frankel, personal communication.

[G] M. Gromov, Topological invariants of dynamical systems and spaces of holomorphic maps. I, Math. Phys. Anal. Geom. 2 (1999), 323-415. Zbl 1160.37322 MR 1742309

[H] L. Hörmander, $L^{2}$ estimates and existence theorems for the $\bar{\partial}$ operator, Acta Math. 113 (1965), 89-152. Zbl 0158.11002 MR 0179443

[K] K. Kodaira, On Kähler varieties of restricted type, Proc. Nat. Acad. Sci. U.S.A. 40 (1954), 313-316. Zbl 0055.15703 MR 0068870

[M] Y. Matsushima, On the intermediate cohomology group of a holomorphic line bundle over a complex torus, Osaka J. Math. 16 (1979), 617-631. Zbl 0422.32024 MR 0551580

[O-1] T. Ohsawa, Pseudoconvex domains in $\mathbb{P}^{n}$ : a question on the 1-convex boundary points, in Analysis and geometry in several complex variables (Katata, 1997), Trends Math., Birkhäuser Boston, Boston, MA, 1999, 239-252. Zbl 0971.32007 MR 1699848

[O-2] _ A generalization of Matsushima's embedding theorem, J. Math. Kyoto Univ. 48 (2008), 383-388. Zbl 1192.32010 MR 2436743

[O-S] T. Ohsawa and N. Sibony, Kähler identity on Levi flat manifolds and application to the embedding, Nagoya Math. J. 158 (2000), 87-93. Z Zbl 0976.32021 MR 1766573

[T] G. Tian, On a set of polarized Kähler metrics on algebraic manifolds, J. Differential Geom. 32 (1990), 99-130. Zbl 0706.53036 MR 1064867 